\title{
Intravascular lipoma of the superior vena cava
}

\author{
Maria Cristina Bravi · Serena Salvadei • \\ Pietro Scarponi - Antonio Loforte • \\ Francesco Musumeci • Laura Gasbarrone
}

Received: 30 January 2011/Accepted: 18 March 2011/Published online: 9 April 2011

(C) SIMI 2011

\section{Case presentation}

A 63-year-old man presented to the emergency department (ED) with left-sided abdomen pain. The patient reported a 5-day history of pain under the right shoulder blade and in the lumbar region, associated with constipation, but not with vomiting, diarrhea or any change in urination. The patient was taking prescribed medications: sennonides and rifaximin that had solved the constipation, but had not completely relieved the abdominal pain. His past medical history was significant for an appendectomy many years prior. He denied having any prior medical problems or allergies. On physical examination, he was oriented, alert, comfortable and in no acute distress. The vital signs were: heart rate of 90 beats/min., blood pressure 130/80 $\mathrm{mmHg}$, respiratory rate 16 breaths $/ \mathrm{min}$, and temperature $35.8^{\circ} \mathrm{C}$. The cardiovascular and respiratory examinations were unremarkable. The abdomen was soft, but the patient had tenderness in the epigastric region and in the right hypochondrium with guarding, and a mildly positive Murphy's sign. No rebound tenderness, tenderness at McBurney's point, palpable masses or abnormal pulsations was noted. Digital rectal examination was normal. On auscultation, normal bowel sounds were heard. The genitourinary examination was normal. Laboratory analysis revealed a lipase level of $225 \mathrm{U} / \mathrm{L}$ (range 6-50 U/L), amylase level of

M. C. Bravi $(\bowtie) \cdot$ S. Salvadei · P. Scarponi · L. Gasbarrone Department of Internal Medicine, S. Camillo-Forlanini Hospital, P.za C. Forlanini n.1, 00151 Rome, Italy

e-mail: mcbravi@tiscali.it

A. Loforte - F. Musumeci

Department of Cardiac Surgery and Transplantation,

S. Camillo-Forlanini Hospital, P.za C. Forlanini n.1,

00151 Rome, Italy
$93 \mathrm{U} / \mathrm{L}$ (range 13-53 U/L) and blood glucose level of $146 \mathrm{mg} / \mathrm{dL}$ (range $70-110 \mathrm{mg} / \mathrm{dL}$ ). The serum blood urea nitrogen, creatinine, liver function tests, albumin, electrolytes, lactate dehydrogenase level and complete blood count (CBC) were normal. Coagulation studies, including prothrombin time, activated partial thromboplastin time and serum fibrinogen, were unremarkable. A chest and abdomen radiographs and an abdominal ultrasonography were performed, but all of them were unrevealing. An electrocardiogram demonstrated rapid atrial fibrillation without any acute injury pattern. A thoracoabdominal computed tomography (CT scan) showed a superior vena caval (SVC) filling defect with a subtotal occlusion that extended into the right atrium, like the suprahepatic IVC, with a dishomogeneous aspect. A partial filling defect was also noted at the level of the portal vein, extended up to the left portal vein and the superior mesenteric vein. The interpretations suggested a significant vascular thrombosis. The CT images also revealed gastric walls' thickening. Anticoagulant therapy was started, and the patient was admitted to an internal medicine ward. Laboratory analysis was ordered to re-evaluate the $\mathrm{CBC}$ and the chemistry panel, to monitor the coagulation profile and the urine analysis, to perform a screening for pro-thrombotic states (with homocysteine blood level test, the protein $\mathrm{C}$ and protein $\mathrm{S}$ test and the test for anti-cardiolipin antibodies) and to control tumor markers (with PSA test and CA 19-9 and CEA tumor markers tests) on the possibility that the thrombosis was a paraneoplastic syndrome. The lipase level was $72 \mathrm{U} / \mathrm{L}$, the gamma-glutamyltranspeptidase (GGT) level was $126 \mathrm{U} / \mathrm{L}$ (range 10-40 U/L), the blood glucose level was $165 \mathrm{mg} / \mathrm{dL}$ and the antithrombin III was $64 \%$ (range $80-130 \%$ ); the remainder of the laboratory investigations were normal. An echocardiogram showed a dilatated right atrium and a filling defect of intermediate 
echogenicity in the final portion of the SVC. The blood flow into the right atrium was maintained, but with higher turbulence because of the partial obstruction. Based on the CT report, the patient underwent an esophagogastroduodenoscopy (EGDS) that revealed only features of mild gastritis confirmed by histological study, while a colonoscopy was unremarkable. Since a tumor possibility could not be totally excluded, a second CT scan was performed: the mass, lying within the lumen of the SVC, was unchanged for morphology and size, with fatty densitometric values (Fig. 1). It extended up from the valvular plane of the right atrium to the subclavian vein orifice for about $13 \mathrm{~cm}$. Surprisingly, the CT scan did not show any signs of IVC and portal vein thrombosis that were present on the previous CT probably due to the effect of the anticoagulant therapy begun several days before. An angiomagnetic resonance imaging (MRI) was performed to confirm the nature of the mass and its intraluminal site. It showed the presence of the intraluminal formation inside the SVC. The lumen was reduced to a tiny marginal slit. The mass showed a uniform signal drop on fat-suppressed sequences: it confirmed the diagnosis of an intravascular lipoma. After cardiac surgery consultation, the patient was considered eligible for a surgical excision of the intracavallipoma. A median sternotomy was performed. Cardiopulmonary bypass was commenced utilising a cannulation of the inferior vena cava and innominate vein for blood drainage, and cannulation of distal ascending aorta for arterial return. When moderate hypothermia was reached,

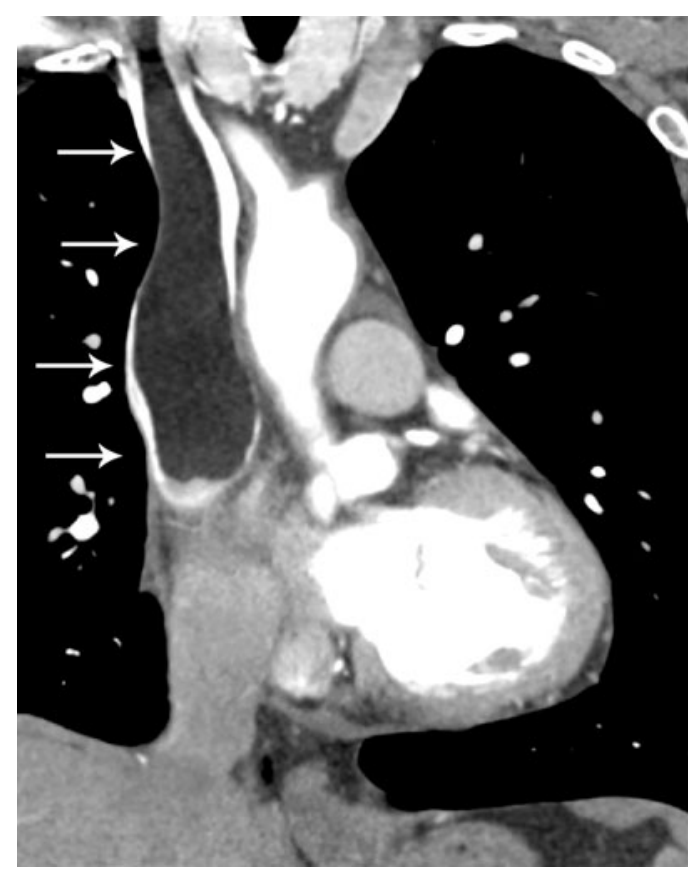

Fig. 1 Contrast-enhanced CT-scan of the chest showing a lesion of fat density within the SVC (arrows)

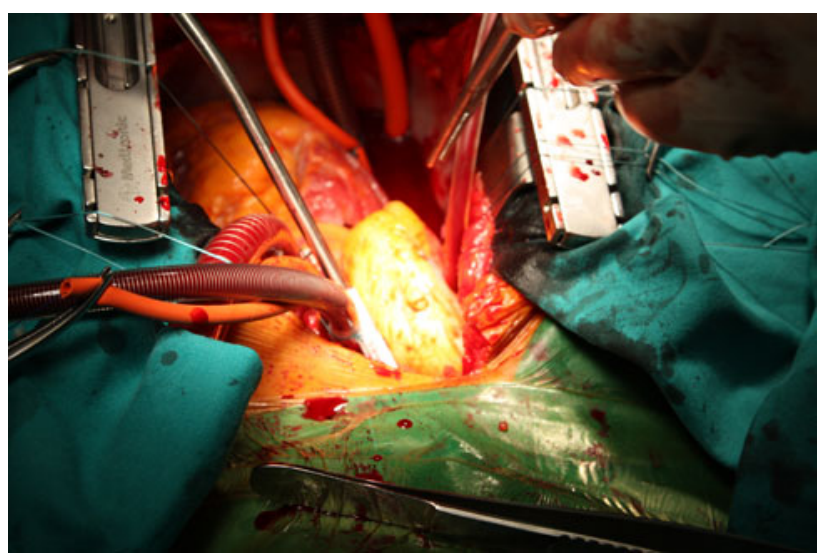

Fig. 2 Surgical removal of the lipoma: the yellowish central mass is identifiable as the tumor inside the SVC

the superior vena cava was incised and opened. The tumor appeared to arise from the distal end of the posterior surface of the superior vena cava just before its bifurcation. The mass was completely removed by pulling it out from the right atrium chamber, and cut distally. The surgical defect was closed by using a pericardial patch (Fig. 2). On macroscopic examination, the mass was soft, lobulated, filled with fat, with a smooth surface, enclosed by a thin capsule; on histological examination, it showed mature adipocytes and collagen.

\section{Discussion}

Primary tumors of the major central veins are rare and the inferior vena cava is the vein more frequently involved. Some cases of a lipoma projecting into the inferior vena cava (IVC), brachiocephalic vein, femoral vein and rarely into the superior vena cava (SVC) have been described previously [1-3]. We report a case of intravascular lipoma of the SVC in a patient without any signs or symptoms directly correlated with the tumor. Usually surgical excision is indicated only when the tumor causes pain or compression of adjacent structures. In this case, the cardiac surgeon decided to operate because of the slowing and turbulent blood flow, and subsequently thrombotic complication in the IVC and venous portal system. The clinical presentation was not clear, and the first evaluations did not lead to a well-defined diagnosis: the patient was not really symptomatic and the examination was not helpful. The CT scan was obtained to exclude pancreatitis because of the high lipase, amylase and blood glucose levels. Unexpectedly, the CT scan showed a significant intravascular thrombosis at the level of the SVC and IVC. Furthermore, the $\mathrm{CT}$ images revealed a suspicious gastric wall thickness, thus according to recent publications [4, 5], a paraneoplastic syndrome was believed to be the most likely 
diagnosis. Therefore, anticoagulant therapy was started. Laboratory analysis was ordered to obtain the tumor markers, and the EGDS and the colonoscopy were performed to exclude malignancy. The only significant examination that suggested the definitive diagnosis was the second CT scan. The anticoagulant therapy was efficacious only in the venous district of the IVC and the portal vein. This suggested that the cause of obstruction of the SVC was of a different nature. A well-executed densitometric study of the CT scan and the further investigation with MRI confirmed the presence of a lipoma. The patient was judged in need of surgical intervention since the lipoma was complicated by the thrombosis of the IVC and the portal vein.

Intravascular lipomas may be asymptomatic, incidentally revealed by imaging, or they may cause a venous obstructing or mediastinal syndromes by virtue of an excessive size causing compressive effects. This reflects the benign nature, the slow growth of the tumor and the compressibility of lipomas in general. In our case, the patient was free of symptoms or signs directly caused by the intracavallipoma. The left-sided abdominal pain, which the patient presented to the ED, may be explainable by the partial obstruction of the suprahepatic IVC and the portal vein, seen in the first contrast-enhanced $\mathrm{CT}$, which both disappeared in the second CT scan after the anticoagulant therapy. Moreover, laboratory analysis during the admission revealed a low antithrombin III, which can be explained by consumption during the thrombotic event.
This confirmed the thrombotic nature of filling defects previously presented in those venous districts. We postulated that the thrombosis was caused by the slowing down of blood flow in the venous districts of the IVC, due to the subtotal occlusion of the SVC. The postoperative period was free from complications, and the patient was discharged with only anticoagulant therapy for the persistent atrial fibrillation. After a follow-up of 3 months, the patient had a completely normal laboratory analysis, and there were no signs or symptoms of recurrences.

Conflict of interest None.

\section{References}

1. Hines J, Katz DS, Goffner L, Rubin GD (1999) Fat collection related to the intrahepatic inferior vena cava on CT. AJR 172:409-411

2. McClure MJ, Sarrazin J, Kapusta L, Murphy J, Arenson AM, Geerts W (2001) Intravascular femoral vein lipoma: an unusual cause of lower limb venous obstruction. AJR 176:463-464

3. Vinnicombe S, Wilson AG, Morgan R, Saunders K (1994) Intravascular lipomas of the superior vena cava: CT features. J Comput Assist Tomogr 18:824-827

4. Zuffa M (2010) Paraneoplastic thromboembolic syndrome as the first sign of a malignant disease. Vnitr Lek 56:49-52

5. Diaconu C, Mateescu D, Bălăceanu A, Marcu M, Jianu V, Stănică A (2010) Pancreatic cancer presenting with paraneoplastic thrombophlebitis-case report. J Med Life 3:96-99 